\title{
miR-137 regulates the migration of human umbilical vein endothelial cells by targeting ephrin-type A receptor 7
}

\author{
YUCHENG LU ${ }^{1}$, XUEYUAN HENG ${ }^{2}$, JIXU YU ${ }^{3}$, QUANPING SU ${ }^{1}$, XIANGHONG GUAN ${ }^{1}$, \\ CUIPING YOU ${ }^{1}$, LONG WANG ${ }^{1}$ and FENGYUAN CHE ${ }^{1,3}$ \\ ${ }^{1}$ Central Laboratory; Departments of ${ }^{2}$ Neurosurgery and ${ }^{3}$ Neurology, Linyi People's Hospital, \\ Shandong University, Linyi, Shandong 276000, P.R. China
}

Received July 3, 2013; Accepted March 4, 2014

DOI: $10.3892 / \mathrm{mmr} .2014 .2319$

\begin{abstract}
MicroRNAs (miRNAs) are short non-coding RNAs, which negatively regulate gene expression. Post-transcriptional regulation by miRNAs is important for organism development. In addition, endothelial cells are key regulators of angiogenesis. By using the 3-(4,5-dimethylthiazol-2-yl)-2,5-diphenyltetrazolium bromide (MTT), migration and gelatin sponge-chorioallantoic membrane assays, it was demonstrated that when miR-137 was overexpressed, cell viability and migration decreased. In addition, it was observed that blocking endogenous miR-137 increased cell viability and migration. Bioinformatics analysis indicated that the 3'-untranslated region (3'UTR) of the ephrin type-A receptor 7 (EPHA7) has a putative binding site for miR-137. miR-137 is able to directly bind to the EPHA7 3'UTR and negatively regulate the expression of EPHA7. miR-137 is also able to decrease the growth and migration of human umbilical vein endothelial cells (HUVECs). The identification of the function of miR-137 and its target gene EPHA7 in HUVECs may provide novel insights into the mechanisms of angiogenesis.
\end{abstract}

\section{Introduction}

Angiogenesis is the process by which novel blood vessels form through the growth of existing blood vessels. It is a complex process involving the proliferation, sprouting and migration of endothelial cells, followed by pruning and remodeling of the vascular network (1). The endothelium is the main regulator of angiogenesis and is highly responsive to factors in the extracellular environment. When the balance of this physiological process is disturbed, it may result in a variety of diseases,

Correspondence to: Professor Fengyuan Che, Department of Neurology, Linyi People's Hospital, Shandong University, 27 Jiefang Road, Linyi, Shandong 276000, P.R. China

E-mail: che1971@126.com

Key words: microRNA-137, angiogenesis, human umbilical vein endothelial cells, ephrin type-A receptor 7 , cell viability, cell migration including cancer, thrombosis, diabetic retinopathy and inflammatory disorders.

MicroRNAs (miRNAs) are a class of conserved non-coding small RNAs, which mainly regulate gene expression post-transcriptionally by targeting the 3'-untranslated regions (3'UTRs) of mRNAs and participate in almost all cellular processes (2). Recently, numerous studies have demonstrated that miRNAs are also important in regulating angiogenesis (3-5). Firstly, it was demonstrated that the miRNA Dicer was important in angiogenesis. The results showed that knocking down Dicer led to the formation of severely compromised embryos and yolk sacs (6). A further study demonstrated that miRNA participates in angiogenesis in succession. Through the detection of miRNA expression levels in human umbilical vein endothelial cells (HUVECs), it was revealed that miR-21, miR-126, miR-221/222, the let-7 family, the miR-17 92 cluster and the miRNA-23 24 cluster were highly expressed in endothelial cells (Ecs) (7-11). Excluding the effects on the phenotype and angiogenesis of ECs, the abnormal expression of certain miRNAs also correlated with tumor angiogenesis, including the miR-17 92 cluster, miR-210, miR-221/222 and miR-296 (6,7,8,9,11-14).

The findings suggest that miRNA expression is required for angiogenesis. At present, although certain miRNAs that affect angiogenesis have been identified, the specific role of miRNAs in angiogenesis remains to be elucidated.

In the present study, it was demonstrated that overexpressing or inhibiting miR-137 was able to regulate the viability and migration of HUVECs by targeting the ephrin type-A receptor 7 (EPHA7). This finding may provide a greater understanding of the process of angiogenesis.

\section{Materials and methods}

Cell culture and transfection. HUVECs were isolated following Nature Protocols (15). Cells were propagated and maintained in minimal essential medium (MEM)- $\alpha$ (Invitrogen Life Technologies, Carlsbad, CA, USA) supplemented with $20 \%$ fetal bovine serum (FBS) and antibiotics [1\% penicillin $(10,000 \mathrm{U} / \mathrm{ml}) /$ streptomycin $(10 \mathrm{mg} / \mathrm{ml})(\mathrm{Gibco}$, Milan, Italy] in a humidified atmosphere at $37^{\circ} \mathrm{C}$ with $5 \% \mathrm{CO}_{2}$. Plasmids or antisense oligonucleotides (ASO) were transfected in antibiotic-free Opti-MEM medium (Invitrogen Life 
A

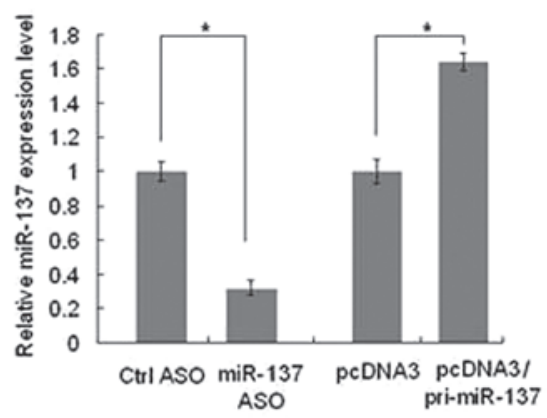

B

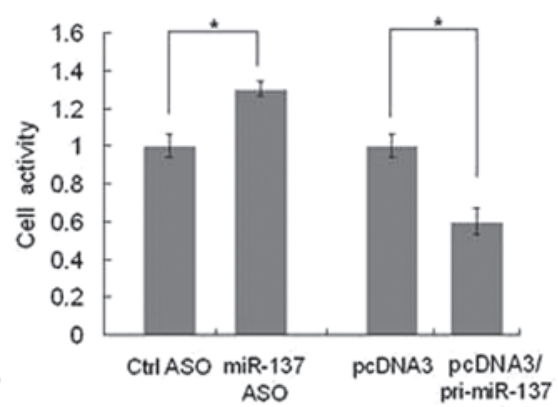

Figure 1. miR-137 decreases the viability of HUVECs. (A) HUVECs were transfected with pcDNA3/pri-miR-137, a control vector, miR-137-ASO or control ASO, respectively. The expression level of miR-137 was detected by quantitative polymerase chain reaction. Expression levels were normalized to the small nuclear RNA U6. (B) Cell viability was measured by the 3-(4,5-dimethylthiazol-2-yl)-2,5-diphenyltetrazolium bromide assay 48 h post-transfection. The histogram shows the mean \pm standard deviation of $\mathrm{A}_{570}$ from three independent experiments. ${ }^{*} \mathrm{P}<0.05$. miR, microRNA; HUVECs, human umbilical vein endothelial cells; ASO, antisense oligonucleotides.

Technologies) with lipofectamine ${ }^{\mathrm{TM}} 2000$ reagent (Invitrogen Life Technologies) according to the manufacturer's instructions.

Vector construction. The miR-137 expression plasmid pcDNA3/pri-miR-137 was constructed. The DNA fragment carrying pre-miR-137 was amplified from genomic DNA by PCR using the following specific primers: miR-137-S, 5'-CG CGGATCCAGCAAGAGTTCTGGTGGC-3'; miR-137-AS, 5'-CCGGAATTCACACCCGAGGA AATGAAAAG-3'. The fragment was then cloned into pcDNA3 between BamHI and EcoRI restriction sites.

To construct the EPHA7 expression vector, the coding sequence of EPHA7 without the 3'UTR was obtained by PCR using the following primers: EPHA7, forward 5'-CGGG ATCCTACCCTAGAAGGAAGAGGTG-3' and reverse 5'-CGGAATTCAATTCTGGGGTAGTTCATG-3'. The 3'UTR of EPHA7 containing the miR-137 binding site was cloned into pcDNA3/enhanced green fluorescent protein (EGFP). Similarly, the fragment of the EPHA7 3'UTR mutant, which contained a triple point mutation in the miR-137 binding site, was also cloned into the pcDNA3/EGFP.

\section{3-(4,5-dimethylthiazol-2-yl)-2,5-diphenyltetrazolium}

bromide (MTT) assay. The MTT assay was used to determine relative cell viability. Cell viability was detected $48 \mathrm{~h}$ after transfection in 96 -well plates. The absorbance at $570 \mathrm{~nm}$ was detected using an IQuant Universal Microplate Spectrophotometer (BioTek Instruments, Inc., Winooski, VT, USA).

Migration assay. In the transwell assays, HUVECs were transfected with pcDNA3/pri-miR-137, a control vector, miR-137-antisense oligonucleotide (ASO) or control ASO, respectively. Following $48 \mathrm{~h}, 4 \times 10^{4}$ cells suspended in serum-free medium were plated onto a gelatin-coated, $8.0-\mu \mathrm{m}$ pore size polycarbonate membrane in 24 -well plates (Corning Inc., Corning, NY, USA). The lower chamber contained MEM- $\alpha$ medium with $20 \%$ FBS and $20 \mathrm{ng} / \mathrm{ml}$ vascular endothelial growth factor (VEGF). Following 6 and $18 \mathrm{~h}$, the migrated cells were fixed with a crystal violet stain and images were captured for counting.
In the scratch assay, once the cells reached $100 \%$ confluence and formed a monolayer, a $200-\mu 1$ pipette tip was used to create a scratch on the cell monolayer. The plate was washed once with $2 \%$ phosphate-buffered saline and replaced with $2 \%$ FBS medium. Migration was quantified by measuring the cell recovery area.

Fluorescent reporter assays. pcDNA3/pri-miR-137, a control vector, miR-137-ASO and control ASO were transfected into HUVECs in 48-well plates, respectively and then with the reporter vector pcDNA3/EGFP-EPHA7-UTR or pcDNA3/EGFP-EPHA7-MUT the following day. The vector pDsRed2-N1 (Clontech Laboratories, Mountain View, CA, USA), expressing red fluorescent protein (RFP), was spiked in and used for normalization. Following $72 \mathrm{~h}$, the cells were lysed with RIPA lysis buffer $(150 \mathrm{mM} \mathrm{NaCl}, 50 \mathrm{mM}$ Tris- $\mathrm{HCl}, \mathrm{pH} 7.2 ; 1 \%$ Triton $\mathrm{X}-100$ and $0.1 \%$ SDS). The intensities of EGFP and RFP fluorescence were detected using a Fluorescence Spectrophotometer F-4500 (Hitachi, Tokyo, Japan).

Western blot analysis. Proteins from transfected HUVECs were extracted $72 \mathrm{~h}$ post-transfection using RIPA buffer and protein expression was analyzed by western blot analysis. GAPDH served as a loading control. The proteins were electrotransferred onto supported nitrocellulose membranes (Amersham, Uppsala, Sweden) by a semi-dry transfer. The membranes were blocked in 5\% skimmed milk in Tris-buffered saline with Tween 20 (TBS-T) containing $0.05 \%$ Tween 20 at room temperature for $2 \mathrm{~h}$, and then incubated at room temperature for $2 \mathrm{~h}$ with antibodies diluted in $1 \%$ skimmed milk in TBS-T, followed by incubating with the appropriate horseradish peroxidase-linked secondary antibodies. The following antibodies were used: rabbit anti-EPHA7, rabbit anti-GAPDH and goat anti-rabbit (Santa Cruz Biotechnology Inc., Santa Cruz, CA, USA).

Gelatin sponge-chorioallantoic membrane (CAM) assay. The chick embryo CAM formed on day 4 of incubation by fusion of the chorion and the allantois. Since it mediates gas exchanges with the extraembryonic environment until hatching, it has a thick capillary network that forms a continuous surface in 
A

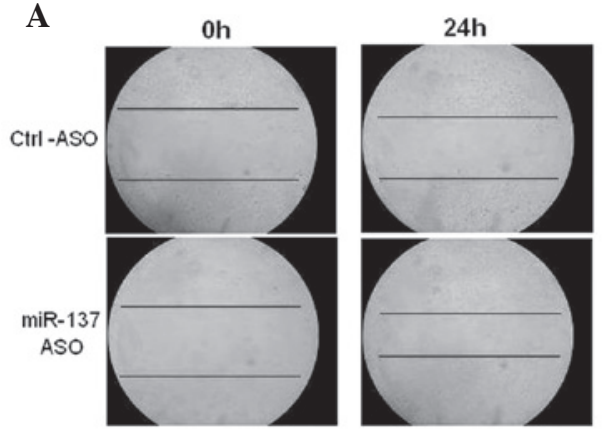

C

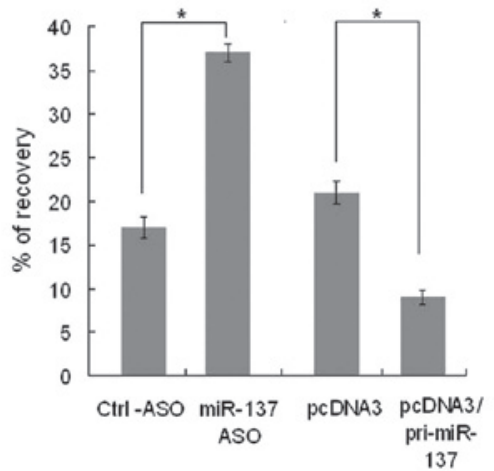

B

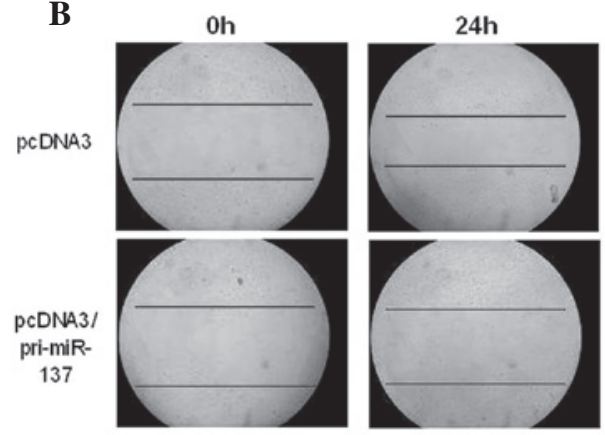

D

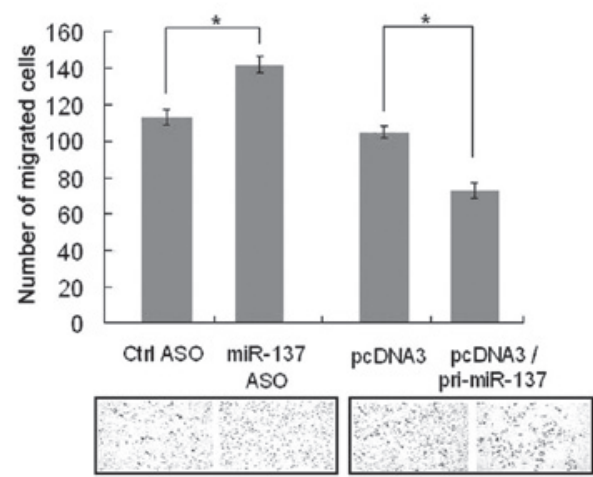

Figure 2. miR-137 suppressed the migration of HUVECs. HUVECs were transfected with pcDNA3/pri-miR-137, a control vector, miR-137-ASO or control ASO. (A and B) In the scratch assay, black lines indicate the width of the wound. (C) The wound areas were calculated and the results shown represent the mean \pm standard deviation from three independent experiments. (D) In the transwell assay, HUVECs were allowed to migrate through filters for 6 and $18 \mathrm{~h}$. Migrated cells were fixed with crystal violet stain and images were captured for counting (magnification, $\mathrm{x} 3,400$ ). ${ }^{*} \mathrm{P}<0.05$. miR, microRNA; HUVECs, human umbilical vein endothelial cells; ASO, antisense oligonucleotides.

A

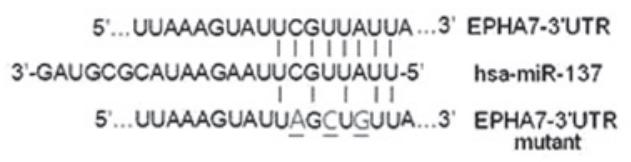

$\mathbf{C}$

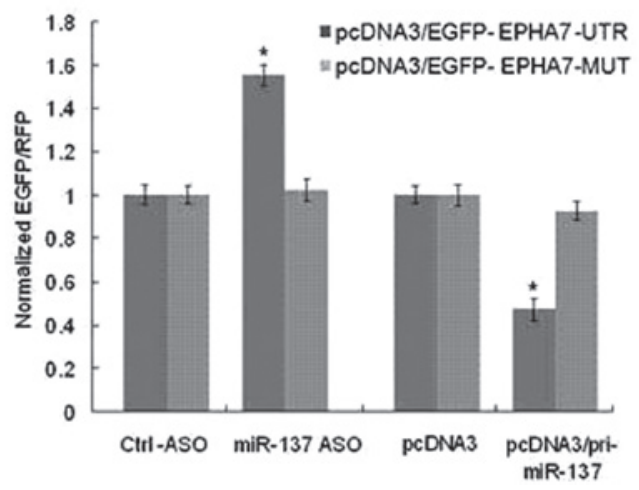

B

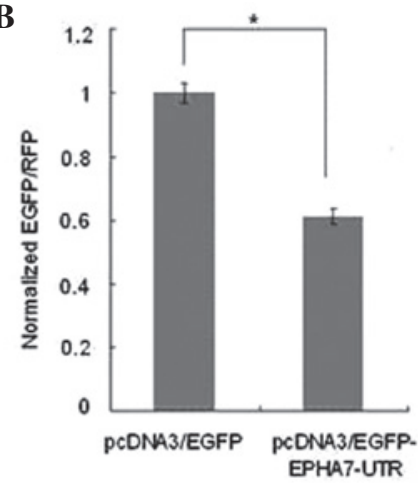

Figure 3. EPHA7 is the target gene of miR-137. (A) As the TargetScan predicted, the EPHA7 3'UTR has a miR-137 binding site. The EPHA7 3'UTR mutant contains a mutant miR-137 binding site. The grey base underlined indicates the mutated nucleotides. (B) HUVECs were transfected with pcDNA3/EGFP or pcDNA3/EGFP-EPHA7-UTR. pDsRed2-N1 expressing RFP was also spiked in for normalization. The fluorescence value in the control group was set to 1. (C) HUVECs were transfected with either pcDNA3/EGFP-EPHA7-UTR reporter vector or pcDNA3/EGFP-EPHA7-MUT mutant vector, along with pcDNA3/pri-miR-137, control vector, miR-137-ASO or control ASO as indicated. The fluorescence value in the control group was set to 1 . "P<0.05. HUVECs, human umbilical vein endothelial cells; ASO, antisense oligonucleotides; EPHA7, ephrin type-A receptor 7; 3'UTR, 3' untranslated region; RFP, red fluorescent protein; EGFP, enhanced green fluorescence protein; miR, microRNA. 
A

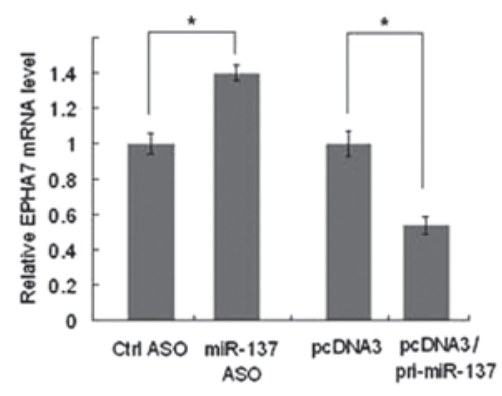

B

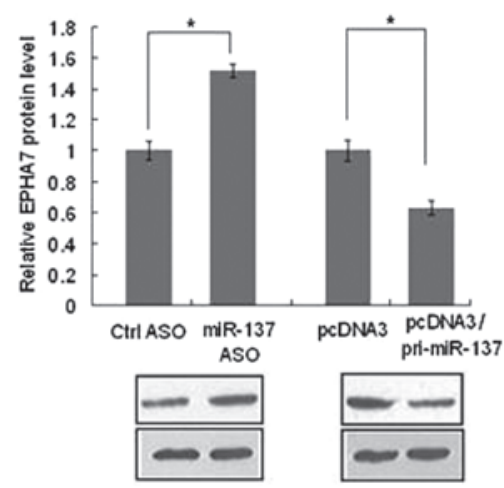

Figure 4. miR-137 negatively regulates the endogenous EPHA7 expression level. (A) RNA was extracted from transfected HUVECs cells and the EPHA7 mRNA level was detected by quantitative polymerase chain reaction. Values were normalized to GAPDH mRNA and the level of EPHA7 mRNA in the control group was set to 1. (B) The protein level of EPHA7 was measured by western blot analysis. ${ }^{*} \mathrm{P}<0.05$. miR, microRNA; HUVECs, human umbilical vein endothelial cells; EPHA7, ephrin type-A receptor 7; ASO, antisense oligonucleotides.

direct contact with the shell. Rapid capillary proliferation continues until day 12; the mitotic index then decreases just as rapidly and the vascular system attains its final arrangement on day 18 , immediately prior to hatching (15).

Statistical analysis. All experiments were repeated a minimum of three times, statistical significance was determined using Student's t-test. In all figures, values are expressed as the mean \pm standard deviation. $\mathrm{P}<0.05$ was considered to indicate a statistically significant difference.

\section{Results}

miR-137 decreases the viability of HUVECs. In order to determine the effects of miR-137 on angiogenesis, HUVECs were used as a model for in vitro study. Plasmids expressing the miR-137 precursor (pcDNA3/pri-miR-137) were constructed and the 2'-Ome miR-137 ASO was synthesized. The efficiency of either overexpression or suppression of miR-137 in HUVECs was validated by quantitative (q) PCR. qPCR results demonstrated that transfection with pcDNA3/pri-miR-137 resulted in a 60\% increase in miR-137 levels in HUVECs, whereas miR-137 2'-Ome ASO resulted in a 70\% reduction in miR-137 levels compared with the control group (Fig. 1A). Transfection efficiency was $>90 \%$, as determined using the pDsRed2-N1 or Cy5-oligomer (data not shown). To explore the effects of human miR-137 on the viability of HUVECs, the MTT assay was used to determine whether miR-137 affects cell viability. As shown in Fig. 1B, when the level of miR-137 was overexpressed, the viability of HUVECs decreased $\sim 40 \%$, whereas when miR-137 was blocked, the cell viability was increased $\sim 30 \%$ compared with the control.

miR-137 suppresses the migration of HUVECs. A transwell assay and scratch assay were used to determine the effects of miR-137 on cell migration (Fig. 2). HUVECs were transfected with pcDNA3/pri-miR-137, a control vector, miR-137-ASO or control ASO. HUVECs were allowed to migrate through gelatin-coated filters $48 \mathrm{~h}$ post-transfection in response to $20 \%$ FBS and $20 \mathrm{ng} / \mathrm{ml}$ VEGF for 6 or $18 \mathrm{~h}$. When transfected with pcDNA3/pri-miR-137, cells were significantly suppressed,
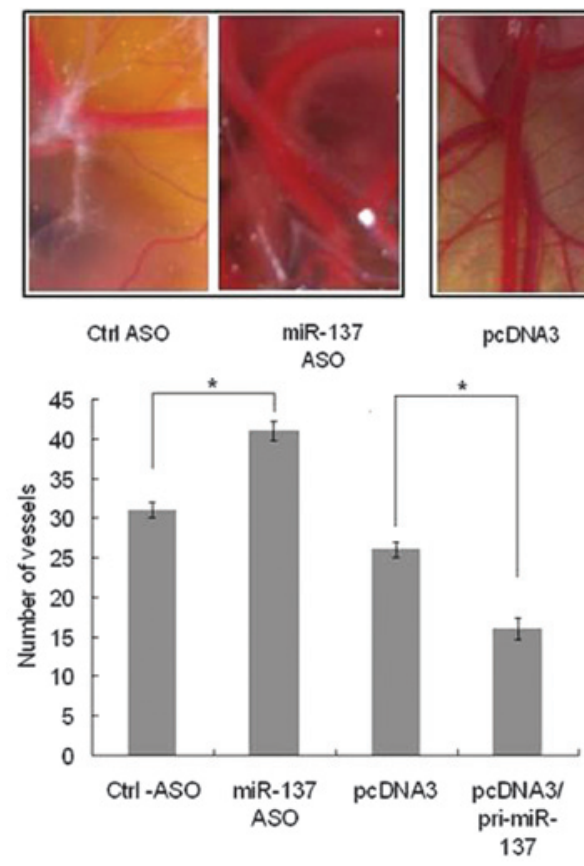

Figure 5. miR-137 inhibits angiogenesis in vivo. Chorioallantoic membrane was transfected with pcDNA3/pri-miR-137, a control vector, miR-137-ASO or control ASO, respectively. After 10 days the number of the vessels were observed and counted. ${ }^{*} \mathrm{P}<0.05$. ASO, antisense oligonucleotides; $\mathrm{miR}$, microRNA.

while blocking miR-137 led to increased cell migration (Fig. 2D). The same results were demonstrated in the scratch assay (Fig. 2A and B). The average recovery percentage of the wounded portion was measured and calculated. When miR-137 was overexpressed, the recovery of the cell area was less than that of the control and when miR-137 was knocked down, the recovery of the cell area was greater than that of the control. (Fig. 2C). These findings indicated that miR-137 suppresses the migration of HUVECs.

EPHA7 is the target gene of miR-137. In the novel miRNA pathway, miRNA produces a marked effect by negatively regulating its target gene. TargetScan was used to predict the 
target gene of miR-137 and it was discovered that EPHA7 has a putative miR-137 binding site in its 3'UTR (Fig. 3A). To confirm whether EPHA7 was the direct target gene of miR-137 and was negatively regulated by it, a vector of the 3'UTR of EPHA7 was constructed and was cloned into the pcDNA3 vector downstream with an EGFP gene. The reporter vector or a pcDNA3/EGFP control vector was transfected into HUVECs. The intensity of EGFP fluorescence in reporter-vector-transfected cells was lower than that in the control group demonstrating that endogenous miRNAs are able to negatively regulate EGFP expression by targeting its 3'UTR (Fig. 3B). HUVECs were then transfected with the reporter vector along with the miR-137 expression vector or ASO. The results demonstrated that ectopic expression of miR-137 was able to reduce the intensity of EGFP fluorescence and blocking miR-137 was able to enhance EGFP expression levels (Fig. 3C). However, when the EPHA7 3'UTR of the miR-137 binding site has three base mutations, the intensity of EGFP fluorescence was not significantly changed by either overexpressing or blocking miR-137 (Fig. 3C). These results suggested that EPHA7 was the direct target gene of miR-137. qPCR and western blot analysis were used to further confirm how miR-137 regulates EPHA7 gene expression. When miR-137 was overexpressed, compared with the control groups, the levels of EPHA7 mRNA (Fig. 4A) and protein (Fig. 4B) decreased by $\sim 35 \%$. Conversely, blocking miR-137 increased the mRNA (Fig. 4A) and protein (Fig. 4B) levels of EPHA7 by $\sim 45 \%$. These results indicate that miR-137 is able to negatively regulate the expression of EPHA7 at a post-transcriptional level, which coincides with the results from the EGFP reporter assay.

miR-137 inhibits angiogenesis in vivo. The gelatin sponge-chorioallantoic membrane assay was used in order to investigate the function of $\mathrm{miR}-137$ in angiogenesis. pcDNA3/pri-miR-137, a control vector, miR-137-ASO or control ASO were transfected in the chick embryo chorioallantoic membrane model according to Nature Protocols (15). Following 10 days, the number of vessels were observed and counted. It was demonstrated that when miR-137 was blocked, the vessels were more abundant than in the control (Fig. 5A), whereas when miR-137 was overexpressed, the vessels were less abundant than i the control (Fig. 5B). This result demonstrated that miR-137 is able to suppress angiogenesis in vivo.

\section{Discussion}

Increasing evidence has indicated that miRNAs may be important in physiological or pathological angiogenesis (3). In the present study, the role of miR-137 in the angiogenic properties of HUVECs was demonstrated. Knockdown of miR-137 was able to significantly increase cell viability and migration, and ectopic expression of miR-137 was able to decrease cell viability and migration. From these results, it was shown that miR-137 is an important antiangiomiR in angiogenesis. Next, EPHA7 was identified to be the direct target gene of miR-137 that may participate in this process. EPHA7 belongs to the Eph/ephrin signaling pathway, which is essential for the patterning of multiple tissues and cell types, including vascular endothelial cell assembly, cell migration, mesenchymal cell condensation, vascular bed formation, tumor neovascularization and the closure of the external genitalia (16-24). EPHA7 is highly conserved in vertebrates and is widely expressed in embryonic tissues, particularly in the developing central nervous system (25). In the present study, it was demonstrated that EPHA7 was negatively regulated by miR-137, and that miR-137 by targeting EPHA7 regulated the migration and angiogenesis of Ecs. It has been demonstrated that miR-137 is able to act as a tumor suppressor in uveal melanoma cell proliferation through the downregulation of MITF and CDK6 (26). Whether miR-137 is able to suppress tumor angiogenesis and thus inhibit tumor growth by targeting EPHA7 remains uncertain and requires further investigation.

In conclusion, the results of the present study indicated that miR-137 targets EPHA7 and regulates the behavior of ECs in vitro and in vivo, including cell viability, migration and angiogenesis. The identification of the antiangiomiR miR-137 and its target gene EPHA7 in HUVECs may aid our understanding of the molecular mechanisms underlying angiogenesis.

\section{References}

1. Carmeliet P: VEGF as a key mediator of angiogenesis in cancer. Oncology 69: 4-10, 2005.

2. Eulalio A, Huntzinger E and Izaurralde, E: Getting to the root of miRNA-mediated gene silencing. Cell 132: 9-14, 2008.

3. Suarez Y and Sessa WC: MicroRNAs as novel regulators of angiogenesis. Circ Res 104: 442-454, 2009.

4. Donnem T, Fenton CG, Lonvik K, Berg T, Eklo K, Andersen S, Stenvold H, AI-Shibli K, AI-Saad S, Bremnes RM and Busund LT: MicroRNA signatures in tumor tissue related to angiogenesis in non-small cell lung cancer. Plos One 7: e29671, 2012.

5. Yamasaki K, Nakasa T, Miyaki S, Yamasaki T, Yasunaqa Y and Ochi M: Angiogenic microRNA-210 is present in cells surrounding osteonecrosis. J Orthop Res 30: 1263-1270, 2012.

6. Yang WJ, Yang DD, Na S, Sandusky GE, Zhang Q and Zhao G: Dicer is required for embryonic angiogenesis during mouse development. J Biol Chem 280: 9330-9335, 2005.

7. Kuehbacher A, Urbich C, Zeiher AM and Dimmeler S: Role of Dicer and Drosha for endothelial microRNA expression and angiogenesis. Circ Res 101: 59-68, 2007.

8. Suárez Y, Fernández-Hernando C, Pober JS and Sessa WC: Dicer dependent microRNAs regulate gene expression and functions in human endothelial cells. Circ Res 100: 1164-1173, 2007.

9. Fasanaro P, D'Alessandra Y, Di Stefano V, Melchionna R, Romani S, Pompilio G, Capogrossi MC and Martelli F: MicroRNA-210 modulates endothelial cell response to hypoxia and inhibits the receptor tyrosine kinase ligand Ephrin-A3. J Biol Chem 283: 15878-15883, 2008.

10. Harris TA, Yamakuchi M, Ferlito M, Mendell JT and Lowenstein CJ: MicroRNA-126 regulates endothelial expression of vascular cell adhesion molecule 1. Proc Natl Acad Sci USA 105: 1516-1521, 2008.

11. Poliseno L, Tuccoli A, Mariani L, Evangelista M, Citti L, Woods K, Mercatanti A, Hammond S and Rainaldi G: MicroRNAs modulate the angiogenic properties of HUVECs. Blood 108: 3068-3071, 2006.

12. Würdinger T, Tannous BA, Saydam O, Skog J, Grau S, Soutschek J, Weissleder R, Breakefield XO and Krichevsky AM: miR-296 regulates growth factor receptor overexpression in angiogenic endothelial cells. Cancer Cell 14: 382-393, 2008.

13. le Sage C, Nagel R, Egan DA, Schrier M, Mesman E, Mangiola A, Anile C, Maira G, Mercatelli N, Ciafrè SA Farace MG and Agami R: Regulation of the p27(Kip1) tumor suppressor by miR-221 and miR-222 promotes cancer cell proliferation. EMBO J 26: 3699-3708, 2007.

14. Bonauer A, Carmona G, Iwasaki M, et al: MicroRNA-92a controls angiogenesis and functional recovery of ischemic tissues in mice. Science 324: 1710-1713, 2009.

15. Ribatti D, Nico B, Vacca A and Presta M: The gelatin sponge-chorioallantoic membrane assay. Nat Protoc 1: 85-91, 2006 . 
16. Wang HU, Chen ZF and Anderson DJ: Molecular distinction and angiogenic interaction between embryonic arteries and veins revealed by ephrin-B2 and its receptor Eph-B4. Cell 93: 741-753, 1998.

17. Ogawa K, Pasqualini R, Lindberg RA, Kain, Freeman AL, Pasquale EB: The ephrin-A1 ligand and its receptor, EphA2, are expressed during tumor neovascularization. Oncogene 19: 6043-6052, 2000.

18. Stadler HS, Higgins KM, Capecchi MR: Loss of Eph-receptor expression correlates with loss of cell adhesion and chondrogenic capacity in Hoxa13 mutant limbs. Development 128: 4177-4188, 2001.

19. Chan J, Mably JD, Serluca FC, Chen JN, Goldstein NB, Thomas MC, Cleary JA, Brennan C, Fishman MC and Roberts TM: Morphogenesis of prechordal plate and notochord requires intact Eph/ephrinB signaling. Dev Bio 234: 470-482, 2001.

20. Dravis C, Yokoyama N, Chumley MJ, Cowan CA, Silvany RE, Shay J, Baker LA and Henkemeyer M: Bidirectional signaling mediated by ephrin-B2 and EphB2 controls urorectal development. Dev Biol 271: 272-290, 2004.

21. Davy A, Aubin J and Soriano P: Ephrin-B1 forward and reverse signaling are required during mouse development. Genes Dev 18: $572-583,2004$.
22. Marquardt T, Shirasaki R, Ghosh S, Andrews SE, Carter N, Hunter T and Pfaff SL: Coexpressed EphA receptors and ephrin-A ligands mediate opposing actions on growth cone navigation from distinct membrane domains. Cell 121: 127-139, 2005.

23. Egea J, Nissen UV, Dufour A, Sahin M, Greer P, Kullander K, Mrsic-Flogel TD, Greenberg ME, Kiehn O, Vanderhaeghen P and Klein R: Regulation of EphA 4 kinase activity is required for a subset of axon guidance decisions suggesting a key role for receptor clustering in Eph function. Neuron 47: 515-528, 2005.

24. Taneja R, Thisse B, Rijli FM, Thisse C, Bouillet P, Chambon P: The expression pattern of the mouse receptor tyrosine kinase gene MDK1 is conserved through evolution and requires Hoxa-2 for rhombomere-specific expression in mouse embryos. Dev Biol 177: 397-412, 1996

25. Ciossek T, Millauer B and Ulrich A: Identification of alternatively spliced mRNAs encoding variants of MDK1, a novel receptor tyrosine kinase expressed in the murine nervous system. Oncogene 10: 97-108, 1995.

26. Chen X, Wang J, Shen H, Lu J, Li C, Hu DN, Dong XD, Yan D and Tu L: Epigenetics, microRNAS, and carcinogenesis: functional role of microRNA-137 in uveal melanoma. Invest Ophthalmol Vis Sci 52: 1193-1199, 2011. 